\title{
PARABOLIC INEQUALITIES IN $L^{1}$ AS LIMITS OF RENORMALIZED EQUATIONS
}

K. AZELMAT, M. KBIRI ALAOUI, D. MESKINE, AND A. SOUISSI

Received 21 April 2006; Revised 10 August 2006; Accepted 21 August 2006

The paper deals with the existence of solutions of some parabolic bilateral problems approximated by the renormalized solutions of some parabolic equations.

Copyright (c) 2006 Hindawi Publishing Corporation. All rights reserved.

\section{Introduction}

Let $\Omega$ be a bounded domain in $\mathbb{R}^{N}$ and $T>0$. We denote by $Q$ the cylinder $\Omega \times(0, T)$ and $\Gamma=\partial Q$.

Let

$$
A(u)=-\operatorname{div}(a(x, t, u, \nabla u))
$$

be a Leray-Lions operator acting on $L^{p}\left(0, T ; W_{0}^{1, p}(\Omega)\right), 1<p<\infty$, into its dual $L^{p^{\prime}}(0, T$; $\left.W^{-1, p^{\prime}}(\Omega)\right)\left(1 / p+1 / p^{\prime}=1\right)$. Consider the following parabolic problem:

$$
\begin{gathered}
u \in \mathscr{K}=\left\{v \in L^{p}\left(0, T ; W_{0}^{1, p}(\Omega)\right): v(t) \in K \text { a.e. }\right\} \\
\int_{0}^{T}\left\langle\frac{\partial v}{\partial t}, u-v\right\rangle d t+\int_{Q} a(x, t, u, \nabla u)(\nabla u-\nabla v) d x d t \leq \int_{0}^{T}\langle f, u-v\rangle d t \\
\forall v \in \mathscr{K} \cap\left\{v \in L^{p}\left(0, T ; W_{0}^{1, p}(\Omega)\right): \frac{\partial v}{\partial t} \in L^{p^{\prime}}\left(0, T ; W^{-1, p^{\prime}}(\Omega)\right) ; v(0)=0\right\},
\end{gathered}
$$

where $K$ is a given convex in $W_{0}^{1, p}(\Omega)$ and $f \in L^{p^{\prime}}\left(0, T ; W^{-1, p^{\prime}}(\Omega)\right)$.

It is well known that $(P)$ admits at least one solution via a classical penalty method (see Lions [5] for $p \geq 2$ and Landes-Mustonen [4] for $1<p<2$ ). Recently in [6], the authors 
approximated $(P)$ by the following sequence of parabolic equations:

$$
\begin{gathered}
\frac{\partial u_{n}}{\partial t}+A\left(u_{n}\right)+\left|h\left(x, u_{n}\right)\right|^{n-1} h\left(x, u_{n}\right)\left|G\left(x, t, u_{n}, \nabla u_{n}\right)\right|=f \quad \text { in } Q \\
u_{n}(x, t)=0 \quad \text { on } \partial Q \\
u_{n}(x, 0)=0 \text { in } \Omega,
\end{gathered}
$$

where $h$ and $G$ are two Carathéodory functions satisfying some natural growth conditions. The obtained convex $K$ depends on two obstacles constructed from $h$.

In the $L^{1}$ case, that is, $f \in L^{1}(\Omega \times] 0, T[)$, the formulations $(P)$ and $\left(P_{n}\right)$ are not appropriate. So, we introduce the renormalized problem $\left(R_{n}\right)$ associated to $\left(P_{n}\right)$ (see the definition below). The study of the asymptotic behavior of $\left(R_{n}\right)$ as $n \rightarrow \infty$ leads to some bilateral parabolic problem. Our approach allows us also to prove the existence of solutions for general parabolic inequalities of type

$$
\begin{aligned}
& T_{k}(u) \in \mathscr{K}, \\
& \int_{0}^{T}\left\langle\frac{\partial v}{\partial t}, T_{k}(u-v)\right\rangle d t+\int_{Q} a(x, t, u, \nabla u) \nabla T_{k}(u-v) d x d t \\
& \quad+\int_{Q} H(x, t, u, \nabla u) T_{k}(u-v) d x d t \leq \int_{Q} f T_{k}(u-v) d x d t, \quad \forall v \in \mathscr{K} \cap D \cap L^{\infty}(Q),
\end{aligned}
$$

where $D=\left\{v \in L^{p}\left(0, T ; W_{0}^{1, p}(\Omega)\right), \partial v / \partial t \in L^{p^{\prime}}\left(0, T, W_{0}^{-1, p^{\prime}}(\Omega)\right)+L^{1}(Q), v(0)=0\right\}$ and where $H$ is a given Carathéodory function satisfying some natural growth assumption.

For some recent and classical results for some parabolic inequalities problems, the reader can refer to $[2,7,9,10]$.

\section{Main result}

Let $\Omega$ be an open bounded subset of $\mathbb{R}^{N}, N \geq 2$ and $1<p<+\infty$.

We denote by $Q$ the cylinder $\Omega \times(0, T)$ and $\Gamma=\partial Q$.

Let $A(u)=-\operatorname{div}(a(x, t, \nabla u))$ be a Leray-Lions operator defined on $L^{p}\left(0, T ; W_{0}^{1, p}(\Omega)\right)$ into its dual $L^{p^{\prime}}\left(0, T ; W^{-1, p^{\prime}}(\Omega)\right)$, where $a: \Omega \times \mathbb{R} \times \mathbb{R}^{N} \rightarrow \mathbb{R}^{N}$ is a Carathéodory function satisfying for a.e. $x \in \Omega$, for all $t \in \mathbb{R}$ and for all $\zeta, \zeta^{\prime} \in \mathbb{R}^{N},\left(\zeta \neq \zeta^{\prime}\right)$ the following hold:

$$
\begin{gathered}
|a(x, t, \zeta)| \leq \beta\left(k(x, t)+|\zeta|^{p-1}\right), \\
\left(a(x, t, \zeta)-a\left(x, t, \zeta^{\prime}\right)\right)\left(\zeta-\zeta^{\prime}\right)>0, \\
a(x, t, \zeta) \zeta \geq \alpha|\zeta|^{p},
\end{gathered}
$$

with $\alpha>0, \beta>0, k \in L^{p^{\prime}}(Q)$.

Furthermore, let $h: \Omega \times \mathbb{R} \rightarrow \mathbb{R}$ be a Carathéodory function such that

$$
h(x, 0)=0, \quad h(x, s) \text { is nondecreasing with respect to } s .
$$


$G$ is a Carathéodory function satisfying the following assumptions:

$$
\begin{aligned}
& |G(x, t, s, \xi)| \leq b(|s|)\left(c(x, t)+|\xi|^{p}\right), \quad G(x, t, s, 0)=0, \\
& \left\{v \in L^{p}\left(0, T ; W_{0}^{1, p}(\Omega)\right): G(x, t, v, \nabla v)=0 \text { a.e. in } Q\right\} \\
& \quad \subset\left\{v \in L^{p}\left(0, T ; W_{0}^{1, p}(\Omega)\right):|h(x, v)| \leq 1 \text { a.e. in } Q\right\} .
\end{aligned}
$$

Let us suppose

for almost $x \in \Omega \backslash \Omega_{+}^{\infty}$ there exists $\epsilon=\epsilon(x)>0$ such that

$$
h(x, s)>1, \quad \forall s \in] q_{+}(x), q_{+}(x)+\epsilon[,
$$

for almost $x \in \Omega \backslash \Omega_{-}^{\infty}$ there exists $\epsilon=\epsilon(x)>0$ such that

$$
h(x, s)<-1, \quad \forall s \in] q_{-}(x)-\epsilon, q_{-}(x)[
$$

where $b$ is a continuous nondecreasing function and $c(x, t) \in L^{1}(Q), c \geq 0$, and

$$
\begin{gathered}
q_{+}(x)=\inf \{s>0, h(x, s) \geq 1\}, \\
q_{-}(x)=\sup \{s>0, h(x, s) \leq-1\}, \\
\Omega_{+}^{\infty}=\left\{x \in \Omega: q_{+}(x)=+\infty\right\}, \\
\Omega_{-}^{\infty}=\left\{x \in \Omega: q_{-}(x)=-\infty\right\} .
\end{gathered}
$$

We define for all $s$ and $k$ in $\mathbb{R}, k \geq 0, T_{k}(s)=\max (-k, \min (k, s))$.

We will say that $u_{n}$ is a renormalized solution of $\left(P_{n}\right)$ if

$$
\begin{gathered}
T_{k}\left(u_{n}\right) \in L^{p}\left(0, T ; W_{0}^{1, p}(\Omega)\right), \quad \forall k>0, \\
\lim _{h \rightarrow \infty} \int_{\left\{h \leq\left|u_{n}\right| \leq h+1\right\}} a\left(x, t, \nabla u_{n}\right) \nabla u_{n} d x d t=0, \\
u_{n} \text { satisfies in the distributional sense } \\
\left(A\left(u_{n}\right)\right)_{t}-\operatorname{div}\left(a\left(x, t, \nabla u_{n}\right) A^{\prime}\left(u_{n}\right)\right)+a\left(x, t, \nabla u_{n}\right) \nabla u_{n} A^{\prime \prime}\left(u_{n}\right) \\
+\left|h\left(x, u_{n}\right)\right|^{n-1} h\left(x, u_{n}\right)\left|G\left(x, t, u_{n}, \nabla u_{n}\right)\right| A^{\prime}\left(u_{n}\right)=f A^{\prime}\left(u_{n}\right),
\end{gathered}
$$

$\forall A \in C^{1}(\mathbb{R}), A^{\prime}, A^{\prime \prime} \in L^{\infty}(\Omega), \quad A^{\prime}$ has a compact support and $u_{n}$ satisfies

the initial condition in the sense that $A\left(u_{n}\right) \in C\left([0, T], L^{1}(\Omega)\right)$.

Thanks to [8, Theorem 3.2, page 164], there exists at least one solution $u_{n}$ of $\left(R_{n}\right)$.

Theorem 2.1. Under the hypotheses (2.1)-(2.5), $f \in L^{1}(Q)$, the problem $\left(P_{n}\right)$ has at least one renormalized solution $\left(u_{n}\right)$ such that

$$
T_{k}\left(u_{n}\right) \longrightarrow T_{k}(u) \text { strongly in } L^{p}\left(0, T ; W_{0}^{1, p}(\Omega)\right) \text {, }
$$


4 Parabolic inequalities in $L^{1}$

where $u$ is a solution of the following obstacle problem:

$$
\begin{gathered}
q_{-}(x) \leq u(x, t) \leq q_{+}(x) \quad \text { a.e. }(x, t) \in Q, \\
T_{k}(u) \in L^{p}\left(0, T ; W_{0}^{1, p}(\Omega)\right), \\
\int_{0}^{T}\left\langle\frac{\partial v}{\partial t}, T_{k}(u-v)\right\rangle d t+\int_{Q} a(x, t, \nabla u) \nabla T_{k}(u-v) d x d t \\
\leq \int_{Q} f T_{k}(u-v) d x d t, \quad \forall v \in \mathscr{K} \cap D \cap L^{\infty}(Q),
\end{gathered}
$$

where

$$
\begin{gathered}
D=\left\{v \in L^{p}\left(0, T ; W_{0}^{1, p}(\Omega)\right), \frac{\partial v}{\partial t} \in L^{p^{\prime}}\left(0, T ; W^{-1, p^{\prime}}(\Omega)\right)+L^{1}(Q), v(0)=0\right\}, \\
\mathscr{K}=\left\{v \in L^{p}\left(0, T ; W_{0}^{1, p}(\Omega)\right), v(t) \in K\right\}, \quad K=\left\{v \in W_{0}^{1, p}(\Omega), q_{-} \leq v \leq q_{+}\right\} .
\end{gathered}
$$

Moreover, if $q_{-}, q_{+} \in L^{\infty}(\Omega)$, then $u \in L^{p}\left(0, T ; W_{0}^{1, p}(\Omega)\right) \cap L^{\infty}(Q)$.

Remark 2.2. The same result can be obtained when dealing with general operator of Leray-Lions type depending also on $u$, that is, $A(u)=-\operatorname{div}(a(x, t, u, \nabla u))$.

Proof of Theorem 2.1.

Step 1. Let $A(t)=H_{m}(t), H_{m}(t)=\int_{0}^{t} h_{m}(s) d s$, where

$$
h_{m}(s)= \begin{cases}1 & \text { if }|s| \leq m \\ \text { affine } & \text { if } m \leq|s| \leq m+1, \\ 0 & \text { if } m+1 \leq|s|\end{cases}
$$

Taking now $T_{k}\left(H_{m}\left(u_{n}\right)\right)$ as test function in $\left(R_{n}\right)$, we obtain

$$
\begin{aligned}
\int_{0}^{T}\left\langle\frac{\partial H_{m}\left(u_{n}\right)}{\partial t}, T_{k}\left(H_{m}\left(u_{n}\right)\right)\right\rangle d t+\int_{\left|H_{m}\left(u_{n}\right)\right|<k} a\left(x, t, \nabla u_{n}\right) \nabla u_{n} h_{m}^{2}\left(u_{n}\right) d x d t \\
\quad+\int_{Q}\left|h\left(x, u_{n}\right)\right|^{n-1} h\left(x, u_{n}\right)\left|G\left(x, t, u_{n}, \nabla u_{n}\right)\right| h_{m}\left(u_{n}\right) T_{k}\left(H_{m}\left(u_{n}\right)\right) d x d t \\
\quad+\int_{Q} a\left(\cdot, t, \nabla u_{n}\right) \nabla u_{n} h_{m}^{\prime}\left(u_{n}\right) T_{k}\left(H_{m}\left(u_{n}\right)\right) d x d t=\int_{Q} f h_{m}\left(u_{n}\right) T_{k}\left(H_{m}\left(u_{n}\right)\right) d x d t .
\end{aligned}
$$

Since

$$
\begin{aligned}
& \int_{0}^{T}\left\langle\frac{\partial H_{m}\left(u_{n}\right)}{\partial t}, T_{k}\left(H_{m}\left(u_{n}\right)\right)\right\rangle d t \\
& \quad=\int_{\Omega}\left(\int_{0}^{H_{m}\left(u_{n}(x, T)\right)} T_{k}(s) d s\right) d x-\int_{\Omega}\left(\int_{0}^{H_{m}\left(u_{n}(x, 0)\right)} T_{k}(s) d s\right) d x
\end{aligned}
$$


and by using the fact that $\int_{\Omega}\left(\int_{0}^{H_{m}\left(u_{n}(x, T)\right)} T_{k}(s) d s\right) \geq 0$, we obtain

$$
\begin{gathered}
\int_{\left\{\left|H_{m}\left(u_{n}\right)\right|<k\right\}} a\left(x, t, \nabla u_{n}\right) \nabla u_{n} h_{m}^{2}\left(u_{n}\right) d x d t \leq C k+\int_{\left\{m \leq\left|u_{n}\right| \leq m+1\right\}} a\left(x, t, \nabla u_{n}\right) \nabla u_{n} d x d t, \\
\int_{Q}\left|h\left(x, u_{n}\right)\right|^{n-1} h\left(x, u_{n}\right)\left|G\left(x, t, u_{n}, \nabla u_{n}\right)\right| h_{m}\left(u_{n}\right) T_{k}\left(H_{m}\left(u_{n}\right)\right) d x d t \\
\leq C k+\int_{\left\{m \leq\left|u_{n}\right| \leq m+1\right\}} a\left(x, t, \nabla u_{n}\right) \nabla u_{n} d x d t .
\end{gathered}
$$

We have $H_{m}(s)$ (resp., $h_{m}(s)$ ) tends to $s$ (resp., to 1 ) as $m$ goes to $+\infty$.

Using Fatou's lemma and the definition of the renormalized solution leads to

$$
\begin{gathered}
\int_{Q}\left|\nabla T_{k}\left(u_{n}\right)\right|^{p} d x d t \leq C k, \\
\int_{Q}\left|h\left(x, u_{n}\right)\right|^{n-1} h\left(x, u_{n}\right)\left|G\left(x, t, u_{n}, \nabla u_{n}\right)\right| T_{k}\left(u_{n}\right) d x d t \leq C k,
\end{gathered}
$$

which gives

$$
\int_{Q}\left|h\left(x, u_{n}\right)\right|^{n}\left|G\left(x, t, u_{n}, \nabla u_{n}\right)\right| \frac{\left|T_{k}\left(u_{n}\right)\right|}{k} d x d t \leq C,
$$

and as $k \rightarrow 0$ we obtain

$$
\int_{Q}\left|h\left(x, u_{n}\right)\right|^{n}\left|G\left(x, t, u_{n}, \nabla u_{n}\right)\right| d x d t \leq C
$$

Choosing now a $C^{2}$ function $\rho_{k}$, such that $\rho_{k}(s)=s$ for $|s| \leq k$ and $2 k \operatorname{sign}(s)$ for $|s|>2 k$, we get

$$
\begin{aligned}
& \left(\rho_{k}\left(u_{n}\right)\right)_{t}-\operatorname{div}\left(a\left(x, t, \nabla u_{n}\right) \rho_{k}^{\prime}\left(u_{n}\right)\right)+a\left(x, t, \nabla u_{n}\right) \nabla u_{n} \rho_{k}{ }^{\prime \prime}\left(u_{n}\right) \\
& \quad+\left|h\left(x, u_{n}\right)\right|^{n-1} h\left(x, u_{n}\right)\left|G\left(x, t, u_{n}, \nabla u_{n}\right)\right| \rho_{k}^{\prime}\left(u_{n}\right)=f \rho_{k}\left(u_{n}\right)
\end{aligned}
$$

We deduce that $\left(\rho_{k}\left(u_{n}\right)\right)_{t}$ is bounded in $L^{1}(Q)+L^{p^{\prime}}\left(0, T ; W^{-1, p^{\prime}}(\Omega)\right)$.

Now thanks to the following result.

Lemma 2.3 [11]. Let $p>1$. If $\left(u_{n}\right)$ is a bounded sequence of $L^{p}\left(0, T ; W_{0}^{1, p}(\Omega)\right)$ such that $\partial u_{n} / \partial t$ is bounded in $L^{1}+L^{p^{\prime}}\left(0, T ; W^{-1, p^{\prime}}(\Omega)\right)$, then $u_{n}$ is relatively compact in $L^{p}(Q)$. 


\section{Parabolic inequalities in $L^{1}$}

We deduce that $\rho_{k}\left(u_{n}\right)$ is relatively compact in $L^{p}(Q)$ and so there exists a measurable function $u$ such that $u_{n} \rightarrow u$ a.e. in $Q$.

Finally, we deduce from $(2.13)$ that $T_{k}\left(u_{n}\right) \rightarrow T_{k}(u)$ weakly in $L^{p}\left(0, T ; W_{0}^{1, p}(\Omega)\right)$, and strongly in $L^{p}(Q)$.

Step 2. We are dealing now with the almost convergence of the gradient.

We have to prove that, for $0<\theta<1$,

$$
\lim _{n \rightarrow \infty} \int_{Q}\left(\left[a\left(x, t, \nabla T_{k}\left(u_{n}\right)\right)-a\left(x, t, \nabla T_{k}(u)\right)\right]\left[\nabla T_{k}\left(u_{n}\right)-\nabla T_{k}(u)\right]\right)^{\theta} d x d t=0 .
$$

Let $\omega \in L^{p}\left(0, T ; W_{0}^{1, p}(\Omega)\right)$, we define for any $\mu>0, \omega_{\mu}$ the time regularization of $\omega$,

$$
\omega_{\mu}(x, t)=\mu \int_{-\infty}^{t} \bar{\omega}(x, s) \exp (\mu(s-t)) d s,
$$

where $\bar{\omega}$ is the zero extension of $\omega$ for $s>T$. Furthermore, $\omega_{\mu}$ satisfies the following properties (see [3]):

$$
\omega_{\mu} \longrightarrow \omega \text { strongly in } L^{p}\left(0, T ; W_{0}^{1, p}(\Omega)\right),
$$

$$
\frac{\partial \omega_{\mu}}{\partial t}=\mu\left(\omega-\omega_{\mu}\right) \quad \text { in the distributional sense. }
$$

Letting $\eta>0$, we obtain

$$
\begin{gathered}
\int_{Q}\left(\left[a\left(x, t, \nabla T_{k}\left(u_{n}\right)\right)-a\left(x, t, \nabla T_{k}(u)\right)\right]\left[\nabla T_{k}\left(u_{n}\right)-\nabla T_{k}(u)\right]\right)^{\theta} \\
\leq C \text { meas }\left\{\left|T_{k}\left(u_{n}\right)-T_{k}(u)_{\mu}\right| \geq \eta\right\}^{1-\theta} \\
+C\left(\int _ { \{ | T _ { k } ( u _ { n } ) - T _ { k } ( u ) _ { \mu } | < \eta \} } \left[a\left(x, t, \nabla T_{k}\left(u_{n}\right)\right)\right.\right. \\
\left.\left.-a\left(x, t, \nabla T_{k}(u)\right)\right]\left[\nabla T_{k}\left(u_{n}\right)-\nabla T_{k}(u)\right]\right)^{\theta} .
\end{gathered}
$$


On the other hand, we have

$$
\begin{aligned}
& \int_{\left\{\left|T_{k}\left(u_{n}\right)-T_{k}(u)_{\mu}\right|<\eta\right\}}\left[a\left(x, t, \nabla T_{k}\left(u_{n}\right)\right)-a\left(x, t, \nabla T_{k}(u)\right)\right]\left[\nabla T_{k}\left(u_{n}\right)-\nabla T_{k}(u)\right] d x d t \\
& \leq \int_{\left\{\left|T_{k}\left(u_{n}\right)-T_{k}(u)_{\mu}\right|<\eta\right\}}\left[a\left(x, t, \nabla T_{k}\left(u_{n}\right)\right)\right. \\
& \left.\quad-a\left(x, t, \nabla T_{k}(u)_{\mu}\right)\right]\left[\nabla T_{k}\left(u_{n}\right)-\nabla T_{k}(u)_{\mu}\right] d x d t \\
& \quad+\int_{\left\{\left|T_{k}\left(u_{n}\right)-T_{k}(u)_{\mu}\right|<\eta\right\}} a\left(x, t, \nabla T_{k}\left(u_{n}\right)\right)\left(\nabla T_{k}(u)_{\mu}-\nabla T_{k}(u)\right) d x d t \\
& \quad+\int_{\left\{\left|T_{k}\left(u_{n}\right)-T_{k}(u)_{\mu}\right|<\eta\right\}}\left[a\left(x, t, \nabla T_{k}(u)_{\mu}\right)-a\left(x, t, \nabla T_{k}(u)\right)\right] \nabla T_{k}\left(u_{n}\right) d x d t \\
& \quad-\int_{\left\{\left|T_{k}\left(u_{n}\right)-T_{k}(u)_{\mu}\right|<\eta\right\}} a\left(x, t, \nabla T_{k}(u)_{\mu}\right) \nabla T_{k}(u)_{\mu} d x d t \\
& \quad+\int_{\left\{\left|T_{k}\left(u_{n}\right)-T_{k}(u)_{\mu}\right|<\eta\right\}} a\left(x, t, \nabla T_{k}(u)\right) \nabla T_{k}(u) d x d t \\
& \leq I_{1}+I_{2}+I_{3}+I_{4}+I_{5} .
\end{aligned}
$$

Take $T_{\eta}\left(H_{m}\left(u_{n}\right)-T_{k}(u)_{\mu}\right)$ as test function in $\left(R_{n}\right)$ with $A(t)=H_{m}(t)$. We obtain

$$
\begin{aligned}
& \int_{0}^{T}\left\langle\frac{\partial H_{m}\left(u_{n}\right)}{\partial t}, T_{\eta}\left(H_{m}\left(u_{n}\right)-T_{k}(u)_{\mu}\right)\right\rangle d t \\
& \quad+\int_{\left\{\left|H_{m}\left(u_{n}\right)-T_{k}(u)_{\mu}\right|<\eta\right\}} a\left(x, t, \nabla u_{n}\right) \nabla u_{n} h_{m}^{2}\left(u_{n}\right)-\nabla T_{k}(u)_{\mu} h_{m}\left(u_{n}\right) d x d t \\
& \quad+\int_{Q}\left|h\left(x, u_{n}\right)\right|^{n-1} h\left(x, u_{n}\right)\left|G\left(x, t, u_{n}, \nabla u_{n}\right)\right| h_{m}\left(u_{n}\right) T_{\eta}\left(H_{m}\left(u_{n}\right)-T_{k}(u)_{\mu}\right) d x d t \\
& \quad+\int_{Q} a\left(x, t, \nabla u_{n}\right) \nabla u_{n} h_{m}^{\prime}\left(u_{n}\right) T_{\eta}\left(H_{m}\left(u_{n}\right)-T_{k}(u)_{\mu}\right) d x d t \\
& \quad=\int_{Q} f h_{m}\left(u_{n}\right) T_{\eta}\left(H_{m}\left(u_{n}\right)-T_{k}(u)_{\mu}\right) d x d t .
\end{aligned}
$$

We have

$$
\begin{aligned}
\int_{0}^{T}\left\langle\frac{\partial H_{m}\left(u_{n}\right)}{\partial t}, T_{\eta}\left(H_{m}\left(u_{n}\right)-T_{k}(u)_{\mu}\right)\right\rangle d t \\
=\int_{0}^{T}\left\langle\frac{\partial H_{m}\left(u_{n}\right)-T_{k}(u)_{\mu}}{\partial t}, T_{k}\left(H_{m}\left(u_{n}\right)-T_{k}(u)_{\mu}\right)\right\rangle d t \\
\quad+\int_{0}^{T}\left\langle\frac{\partial T_{k}(u)_{\mu}}{\partial t}, T_{k}\left(H_{m}\left(u_{n}\right)-T_{k}(u)_{\mu}\right)\right\rangle d t
\end{aligned}
$$


8 Parabolic inequalities in $L^{1}$

Using the fact that

$$
\begin{aligned}
& \int_{0}^{T}\left\langle\frac{\partial H_{m}\left(u_{n}\right)-T_{k}(u)_{\mu}}{\partial t}, T_{\eta}\left(H_{m}\left(u_{n}\right)-T_{k}(u)_{\mu}\right)\right\rangle d t \geq 0 \\
& \int_{0}^{T}\left\langle\frac{\partial T_{k}(u)_{\mu}}{\partial t}, T_{k}\left(H_{m}\left(u_{n}\right)-T_{k}(u)_{\mu}\right)\right\rangle d t \\
& \quad=\mu \int_{Q}\left(T_{k}(u)-T_{k}(u)_{\mu}\right) T_{\eta}\left(H_{m}\left(u_{n}\right)-T_{k}(u)_{\mu}\right) d x d t
\end{aligned}
$$

consequently,

$$
\begin{aligned}
& \underset{n \rightarrow \infty}{\limsup } \limsup _{m \rightarrow \infty} \int_{0}^{T}\left\langle\frac{\partial H_{m}\left(u_{n}\right)}{\partial t}, T_{\eta}\left(H_{m}\left(u_{n}\right)-T_{k}(u)_{\mu}\right)\right\rangle d t \\
& \geq \mu \int_{Q}\left(T_{k}(u)-T_{k}(u)_{\mu}\right) T_{\eta}\left(u-T_{k}(u)_{\mu}\right) d x d t=\epsilon(m, n) \geq 0 .
\end{aligned}
$$

This implies that

$$
\begin{aligned}
& \int_{\left\{\left|H_{m}\left(u_{n}\right)-T_{k}(u)_{\mu}\right|<\eta\right\}} a\left(x, t, \nabla u_{n}\right) \nabla u_{n} h_{m}^{2}\left(u_{n}\right)-\nabla T_{k}(u)_{\mu} h_{m}\left(u_{n}\right) d x d t \\
& \quad+\int_{Q}\left|h\left(x, u_{n}\right)\right|^{n-1} h\left(x, u_{n}\right)\left|G\left(x, t, u_{n}, \nabla u_{n}\right)\right| h_{m}\left(u_{n}\right) T_{\eta}\left(H_{m}\left(u_{n}\right)-T_{k}(u)_{\mu}\right) d x d t \\
& \quad+\int_{Q} a\left(x, t, \nabla u_{n}\right) \nabla u_{n} h_{m}^{\prime}\left(u_{n}\right) T_{\eta}\left(H_{m}\left(u_{n}\right)-T_{k}(u)_{\mu}\right) d x d t \\
& \leq \int_{Q} f h_{m}\left(u_{n}\right) T_{\eta}\left(H_{m}\left(u_{n}\right)-T_{k}(u)_{\mu}\right) d x d t+\epsilon(m, n),
\end{aligned}
$$

which gives by using the fact that

$$
\begin{aligned}
& \int_{Q}\left|h\left(x, u_{n}\right)\right|^{n-1} h\left(x, u_{n}\right)\left|G\left(x, t, u_{n}, \nabla u_{n}\right)\right| h_{m}\left(u_{n}\right) T_{\eta}\left(H_{m}\left(u_{n}\right)-T_{k}(u)_{\mu}\right) d x d t \leq C \eta, \\
& \int_{\left\{\left|H_{m}\left(u_{n}\right)-T_{k}(u)_{\mu}\right|<\eta\right\}} a\left(x, t, \nabla u_{n}\right) \nabla u_{n} h_{m}^{2}\left(u_{n}\right)-\nabla T_{k}(u)_{\mu} h_{m}\left(u_{n}\right) d x d t \\
& \leq C \eta+\epsilon(m, n)+\eta \int_{\left\{m \leq\left|u_{n}\right| \leq m+1\right\}} a\left(x, t, \nabla u_{n}\right) \nabla u_{n} d x d t,
\end{aligned}
$$

which gives as $m \rightarrow \infty$,

$$
\int_{\left\{\left|u_{n}-T_{k}(u)_{\mu}\right|<\eta\right\}} a\left(x, t, \nabla u_{n}\right) \nabla u_{n}-\nabla T_{k}(u)_{\mu} d x d t \leq C \eta+\epsilon(n) .
$$


Finally from (2.22),

$$
\left|I_{1}\right| \leq C \eta+\epsilon(n)-\int_{\left\{\left|T_{k}\left(u_{n}\right)-T_{k}(u)_{\mu}\right|<\eta\right\}} a\left(x, t, \nabla T_{k}(u)_{\mu}\right)\left(\nabla T_{k}\left(u_{n}\right)-\nabla T_{k}(u)\right) .
$$

Since $a\left(x, t, \nabla T_{k}(u)_{\mu}\right) \chi_{\left\{\left|T_{k}\left(u_{n}\right)-T_{k}(u)_{\mu}\right|<\eta\right\}} \rightarrow a\left(x, t, \nabla T_{k}(u)_{\mu}\right) \chi_{\left\{\left|T_{k}(u)-T_{k}(u)_{\mu}\right|<\eta\right\}}$ in $L^{p^{\prime}}(Q)$ and $T_{k}\left(u_{n}\right) \rightarrow T_{k}(u)$ weakly in $L^{p}\left(0, T ; W_{0}^{1, p}(\Omega)\right)$, then

$$
\begin{aligned}
& -\int_{\left\{\left|T_{k}\left(u_{n}\right)-T_{k}(u)_{\mu}\right|<\eta\right\}} a\left(x, t, \nabla T_{k}(u)_{\mu}\right)\left(\nabla T_{k}\left(u_{n}\right)-\nabla T_{k}(u)\right) d x d t \\
& \quad=-\int_{\left\{\left|T_{k}(u)-T_{k}(u)_{\mu}\right|<\eta\right\}} a\left(x, t, \nabla T_{k}(u)_{\mu}\right)\left(\nabla T_{k}(u)-\nabla T_{k}(u)\right) d x d t+\epsilon(n) .
\end{aligned}
$$

So

$$
\left|I_{1}\right| \leq C \eta+\epsilon(n) .
$$

For what concerns the term $I_{2}$, one has

$$
I_{2}=\epsilon(n, \mu)
$$

since

$$
\begin{gathered}
a\left(x, t, \nabla T_{k}\left(u_{n}\right)\right) \chi_{\left\{\left|T_{k}\left(u_{n}\right)-T_{k}(u)_{\mu}\right|<\eta\right\}} \longrightarrow a\left(x, t, \nabla T_{k}(u)\right) \chi_{\left\{\left|T_{k}(u)-T_{k}(u)_{\mu}\right|<\eta\right\}} \quad \text { in }\left(L^{p^{\prime}}(Q)\right)^{N}, \\
\left(\nabla T_{k}(u)_{\mu}-\nabla T_{k}(u)\right) \chi_{\left\{\left|T_{k}\left(u_{n}\right)-T_{k}(u)_{\mu}\right|<\eta\right\}} \longrightarrow\left(\nabla T_{k}(u)_{\mu}-\nabla T_{k}(u)\right) \chi_{\left\{\left|T_{k}(u)-T_{k}(u)_{\mu}\right|<\eta\right\}} .
\end{gathered}
$$

In the same way, we show that

$$
I_{3}=\epsilon(n, \mu), \quad I_{4}=\epsilon(n, \mu), \quad I_{5}=\epsilon(n, \mu) .
$$

Combining the above estimates, we get

$$
\lim _{n \rightarrow \infty} \int_{Q}\left(\left[a\left(x, t, \nabla T_{k}\left(u_{n}\right)\right)-a\left(x, t, \nabla T_{k}(u)\right)\right]\left[\nabla T_{k}\left(u_{n}\right)-\nabla T_{k}(u)\right]\right)^{\theta} d x d t=0 .
$$

Then there exists a subsequence also denoted by $\left(u_{n}\right)$ such that

$$
\nabla u_{n} \longrightarrow \nabla u \quad \text { a.e. in } Q \text {. }
$$

Step 3. From (2.16), we deduce that

$$
\int_{Q}\left|h\left(x, u_{n}\right)\right|^{n}\left|G\left(x, t, u_{n}, \nabla u_{n}\right)\right| d x d t \leq C,
$$

which gives for every $\beta>0$,

$$
\int_{\left|h\left(x, T_{\beta}\left(u_{n}\right)\right)\right|>k}\left|G\left(x, t, T_{\beta}\left(u_{n}\right), \nabla T_{\beta}\left(u_{n}\right)\right)\right| d x d t \leq \frac{C}{k^{n}},
$$


where $k>1$. Letting $n \rightarrow+\infty$ for $k$ fixed, we deduce by using Fatou's lemma

$$
\int_{\left|h\left(x, T_{\beta}(u)\right)\right|>k}\left|G\left(x, t, T_{\beta}(u), \nabla T_{\beta}(u)\right)\right| d x d t=0,
$$

and so, by (2.4)

$$
\left|h\left(x, T_{\beta}(u)\right)\right| \leq 1 \quad \text { a.e. in } Q \text {. }
$$

So

$$
q_{-}(x) \leq T_{\beta}(u(x)) \leq q_{+}(x) \text { a.e. in } Q \text {. }
$$

Letting now $\beta \rightarrow+\infty$, we deduce also that

$$
q_{-}(x) \leq u(x) \leq q_{+}(x) \quad \text { a.e. in } Q \text {. }
$$

Step 4. Strong convergence of the truncations.

We will prove that

$$
\lim _{n \rightarrow \infty} \int_{Q}\left[a\left(x, t, \nabla T_{k}\left(u_{n}\right)\right)-a\left(x, t, \nabla T_{k}(u)\right)\right]\left[\nabla T_{k}\left(u_{n}\right)-\nabla T_{k}(u)\right] d x d t=0 .
$$

Fix $k>0$ and let $\varphi(s)=\exp \left(\delta s^{2}\right), \delta>0$. Let $l>k$ and define the function $R_{l}(s)=\int_{0}^{s} \rho_{l}(t) d t$. Let us consider $\omega_{\mu}^{m}=T_{k}\left(H_{m}(u)_{\mu}\right)$, where $v_{\mu}$ is the mollification with respect to time $v$. Letting $v_{\mu}^{m, n}=\rho_{l}\left(H_{m}\left(u_{n}\right)\right) \varphi\left(T_{k}\left(H_{m}\left(u_{n}\right)\right)-\omega_{\mu}^{m}\right)$ as test function in the problem $\left(R_{n}\right)$, we get

$$
\begin{aligned}
\int_{0}^{T}\left\langle\frac{\partial H_{m}\left(u_{n}\right)}{\partial t}, \rho_{l}\left(H_{m}\left(u_{n}\right)\right) \varphi\left(T_{k}\left(H_{m}\left(u_{n}\right)\right)-\omega_{\mu}^{m}\right)\right\rangle d t \\
\quad+\int_{Q} a\left(x, t, \nabla u_{n}\right) \nabla u_{n} h^{2}\left(u_{n}\right) \rho_{l}^{\prime}\left(H_{m}\left(u_{n}\right)\right) \varphi\left(T_{k}\left(H_{m}\left(u_{n}\right)\right)-\omega_{\mu}^{m}\right) d x d t \\
\quad+\int_{Q} a\left(x, t, \nabla u_{n}\right)\left(\nabla T_{k}\left(H_{m}\left(u_{n}\right)\right)-\nabla \omega_{\mu}^{m}\right) \\
\quad \times h_{m}\left(u_{n}\right) \rho_{l}\left(H_{m}\left(u_{n}\right)\right) \varphi^{\prime}\left(T_{k}\left(H_{m}\left(u_{n}\right)\right)-\omega_{\mu}^{m}\right) d x d t \\
\quad+\int_{Q} a\left(x, t, \nabla u_{n}\right) \nabla u_{n} h_{m}^{\prime}\left(u_{n}\right) \rho_{l}\left(H_{m}\left(u_{n}\right)\right) \varphi\left(T_{k}\left(H_{m}\left(u_{n}\right)\right)-\omega_{\mu}^{m}\right) d x d t \\
\quad+\int_{Q}\left|h\left(x, u_{n}\right)\right|^{n-1} h\left(x, u_{n}\right)\left|G\left(x, t, u_{n}, \nabla u_{n}\right)\right| \\
\quad \times h_{m}\left(u_{n}\right) \rho_{l}\left(H_{m}\left(u_{n}\right)\right) \varphi\left(T_{k}\left(H_{m}\left(u_{n}\right)\right)-\omega_{\mu}^{m}\right) d x d t \\
=\int_{Q} f v_{\mu}^{m, n} h_{m}\left(u_{n}\right) d x d t .
\end{aligned}
$$

We deal now with the estimate of each term of the last equalities. 
K. Azelmat et al. 11

Since $H_{m}\left(u_{n}\right) \in L^{p}\left(0, T ; W_{0}^{1, p}(\Omega)\right)$ and $\partial H_{m}\left(u_{n}\right) / \partial t \in L^{p^{\prime}}\left(0, T ; W^{-1, p^{\prime}}(\Omega)\right)+L^{1}(Q)$, there exists a smooth function $H_{m}\left(u_{n}\right)_{\sigma}$ such that as $\sigma \rightarrow 0$,

$$
\begin{gathered}
H_{m}\left(u_{n}\right)_{\sigma} \longrightarrow H_{m}\left(u_{n}\right) \quad \text { strongly in } L^{p}\left(0, T ; W_{0}^{1, p}(\Omega)\right), \\
\frac{\partial H_{m}\left(u_{n}\right)_{\sigma}}{\partial t} \longrightarrow \frac{\partial H_{m}\left(u_{n}\right)}{\partial t} \quad \text { strongly in } L^{p^{\prime}}\left(0, T ; W^{-1, p^{\prime}}(\Omega)\right)+L^{1}(Q) .
\end{gathered}
$$

This implies that

$$
\begin{aligned}
I= & \int_{0}^{T}\left\langle\frac{\partial H_{m}\left(u_{n}\right)}{\partial t}, \rho_{l}\left(H_{m}\left(u_{n}\right)\right) \varphi\left(T_{k}\left(H_{m}\left(u_{n}\right)\right)-\omega_{\mu}^{m}\right)\right\rangle d t \\
= & \lim _{\sigma \rightarrow 0^{+}} \int_{Q}\left(H_{m}\left(u_{n}\right)_{\sigma}\right)^{\prime} \rho_{l}\left(H_{m}\left(u_{n}\right)_{\sigma}\right) \varphi\left(T_{k}\left(H_{m}\left(u_{n}\right)_{\sigma}\right)-\omega_{\mu}^{m}\right) d x d t \\
= & \lim _{\sigma \rightarrow 0^{+}} \int_{Q}\left[R_{l}\left(H_{m}\left(u_{n}\right)_{\sigma}\right)\right]^{\prime} \varphi\left(T_{k}\left(H_{m}\left(u_{n}\right)_{\sigma}\right)-\omega_{\mu}^{m}\right) d x d t \\
= & \lim _{\sigma \rightarrow 0^{+}} \int_{Q}\left[R_{l}\left(H_{m}\left(u_{n}\right)_{\sigma}\right)-T_{k}\left(H_{m}\left(u_{n}\right)_{\sigma}\right)\right]^{\prime} \varphi\left(T_{k}\left(H_{m}\left(u_{n}\right)_{\sigma}\right)-\omega_{\mu}^{m}\right) d x d t \\
& +\int_{Q}\left[T_{k}\left(H_{m}\left(u_{n}\right)_{\sigma}\right)\right]^{\prime} \varphi\left(T_{k}\left(H_{m}\left(u_{n}\right)_{\sigma}\right)-\omega_{\mu}^{m}\right) d x d t \\
= & \lim _{\sigma \rightarrow 0^{+}}\left\{\int_{\Omega}\left[R_{l}\left(H_{m}\left(u_{n}\right)_{\sigma}\right)-T_{k}\left(H_{m}\left(u_{n}\right)_{\sigma}\right) \varphi\left(T_{k}\left(H_{m}\left(u_{n}\right)_{\sigma}\right)-\omega_{\mu}^{m}\right)\right]_{0}^{T} d x d t\right. \\
& \quad-\int_{Q}\left[R_{l}\left(H_{m}\left(u_{n}\right)_{\sigma}\right)-T_{k}\left(H_{m}\left(u_{n}\right)_{\sigma}\right)\right]^{\prime} \varphi^{\prime}\left(T_{k}\left(H_{m}\left(u_{n}\right)_{\sigma}\right)-\omega_{\mu}^{m}\right)\left(T_{k}\left(H_{m}\left(u_{n}\right)_{\sigma}\right)\right. \\
= & \lim _{\sigma \rightarrow 0^{+}}\left\{I_{1}(\sigma)+I_{2}(\sigma)+I_{3}(\sigma)\right\} . \\
& \left.\left.\quad-\omega_{\mu}^{m}\right)^{\prime} d x d t+\int_{Q}\left[T_{k}\left(H_{m}\left(u_{n}\right)_{\sigma}\right)\right]^{\prime} \varphi\left(T_{k}\left(H_{m}\left(u_{n}\right)_{\sigma}\right)-\omega_{\mu}^{m}\right) d x d t\right\}
\end{aligned}
$$

Observe that for $|s| \leq k$ we have $R_{l}(s)=T_{k}(s)=s$ and for $|s|>k$ we have $\left|R_{l}(s)\right| \geq\left|T_{k}(s)\right|$ and, since both $R_{l}(s)$ and $T_{k}(s)$ have the same sign of $s$, we deduce that $\operatorname{sign}(s)\left(R_{l}(s)-\right.$ $\left.T_{k}(s)\right) \geq 0$. Consequently,

$$
I_{1}(\sigma)=\int_{\left\{\left|H_{m}\left(u_{n}\right)_{\sigma}\right|>k\right\}}\left[R_{l}\left(H_{m}\left(u_{n}\right)_{\sigma}\right)-T_{k}\left(H_{m}\left(u_{n}\right)_{\sigma}\right) \varphi\left(T_{k}\left(H_{m}\left(u_{n}\right)_{\sigma}\right)-\omega_{\mu}^{m}\right)\right]_{0}^{T} d x d t \geq 0 .
$$


We have, since $\left(R_{l}(s)-T_{k}(s)\right)\left(T_{k}(s)\right)^{\prime}=0$, for all $s$,

$$
\begin{gathered}
I_{2}(\sigma)=\int_{\left\{\left|H_{m}\left(u_{n}\right)_{\sigma}\right|>k\right\}}\left[R_{l}\left(H_{m}\left(u_{n}\right)_{\sigma}\right)-T_{k}\left(H_{m}\left(u_{n}\right)_{\sigma}\right) \varphi^{\prime}\left(T_{k}\left(H_{m}\left(u_{n}\right)_{\sigma}\right)\right.\right. \\
\left.\left.-\omega_{\mu}^{m}\right)\right]\left(\omega_{\mu}^{m}\right)^{\prime} d x d t \\
=\mu \int_{\left\{\left|H_{m}\left(u_{n}\right)_{\sigma}\right|>k\right\}}\left[R_{l}\left(H_{m}\left(u_{n}\right)_{\sigma}\right)-T_{k}\left(H_{m}\left(u_{n}\right)_{\sigma}\right) \varphi^{\prime}\left(T_{k}\left(H_{m}\left(u_{n}\right)_{\sigma}\right)\right.\right. \\
\left.\left.-\omega_{\mu}^{m}\right)\right]\left(T_{k}\left(H_{m}\left(u_{n}\right)_{\sigma}\right)-\omega_{\mu}^{m}\right) d x d t,
\end{gathered}
$$

by using the fact that $\varphi^{\prime} \geq 0$ and that

$$
\left(R_{l}\left(H_{m}\left(u_{n}\right)_{\sigma}\right)-T_{k}\left(H_{m}\left(u_{n}\right)_{\sigma}\right)\right)\left(T_{k}\left(H_{m}\left(u_{n}\right)_{\sigma}\right)-\omega_{\mu}^{m}\right) \chi_{\left\{\left|H_{m}\left(u_{n}\right)_{\sigma}\right|>k\right\}} \geq 0
$$

the last integral is of the form $\epsilon(m, n)$. We deduce that

$$
\lim _{\sigma \rightarrow 0^{+}} \sup I_{2}(\sigma) \geq \epsilon(m, n)
$$

For $I_{3}(\sigma)$, one has

$$
\begin{aligned}
I_{3}(\sigma)= & \int_{Q}\left[T_{k}\left(H_{m}\left(u_{n}\right)_{\sigma}\right)\right]^{\prime} \varphi\left(T_{k}\left(H_{m}\left(u_{n}\right)_{\sigma}\right)-\omega_{\mu}^{m}\right) d x d t \\
= & \int_{Q}\left[T_{k}\left(H_{m}\left(u_{n}\right)_{\sigma}\right)-\omega_{\mu}^{m}\right]^{\prime} \varphi\left(T_{k}\left(H_{m}\left(u_{n}\right)_{\sigma}\right)-\omega_{\mu}^{m}\right) d x d t \\
& +\int_{Q}\left(\omega_{\mu}^{m}\right)^{\prime} \varphi\left(T_{k}\left(H_{m}\left(u_{n}\right)_{\sigma}\right)-\omega_{\mu}^{m}\right) d x d t .
\end{aligned}
$$

Let $\Phi(s)=\int_{0}^{s} \varphi(t) d t$. Remark that $\left(T_{k}\left(H_{m}\left(u_{n}\right)_{\sigma}\right)-\omega_{\mu}^{m}\right) \varphi\left(T_{k}\left(H_{m}\left(u_{n}\right)_{\sigma}\right)-\omega_{\mu}^{m}\right) \geq 0$.

Integrating by parts, using the fact that $\Phi \geq 0$, and following the same way as above, we have

$$
\lim _{\sigma \rightarrow 0^{+}} \sup _{3}(\sigma) \geq \epsilon(m, n)
$$

Combining these estimates, we conclude that

$$
\int_{0}^{T}\left\langle\frac{\partial H_{m}\left(u_{n}\right)}{\partial t}, \rho_{l}\left(H_{m}\left(u_{n}\right)\right) \varphi\left(T_{k}\left(H_{m}\left(u_{n}\right)\right)-\omega_{\mu}^{m}\right)\right\rangle d t \geq \epsilon(m, n)
$$


We set

$$
I_{4}(m)=\int_{Q}\left|h\left(x, u_{n}\right)\right|^{n-1} h\left(x, u_{n}\right) \rho_{l}\left(H_{m}\left(u_{n}\right)\right) \varphi\left(T_{k}\left(H_{m}\left(u_{n}\right)_{\sigma}\right)-\omega_{\mu}^{m}\right)\left|G\left(x, t, u_{n}, \nabla u_{n}\right)\right|,
$$

so we have

$$
\limsup _{m \rightarrow \infty} I_{4}(m) \geq I_{4}^{1}+I_{4}^{2}
$$

where

$$
\begin{aligned}
& I_{4}^{1}=\int_{\left\{\left|u_{n}\right|<k, 0 \leq u_{n} \leq T_{k}(u)_{\mu}\right\}} \\
& \quad \quad \times\left|h\left(x, u_{n}\right)\right|^{n-1} h\left(x, u_{n}\right) \varphi\left(T_{k}\left(u_{n}\right)-T_{k}(u)_{\mu}\right) \rho_{l}\left(u_{n}\right)\left|G\left(x, t, u_{n}, \nabla u_{n}\right)\right| d x d t, \\
& \quad I_{4}^{2}=\int_{\left\{\left|u_{n}\right|<k, T_{k}(u)_{\mu} \leq u_{n} \leq 0\right\}} \\
& \quad \times\left|h\left(x, u_{n}\right)\right|^{n-1} h\left(x, u_{n}\right) \varphi\left(T_{k}\left(u_{n}\right)-T_{k}(u)_{\mu}\right) \rho_{l}\left(u_{n}\right)\left|G\left(x, t, u_{n}, \nabla u_{n}\right)\right| d x d t .
\end{aligned}
$$

Since $q_{-} \leq T_{k}(u)_{\mu} \leq q_{+}\left(\right.$recall that $\left.q_{-} \leq T_{k}(u) \leq q_{+}\right)$and $0 \leq \rho_{l}\left(u_{n}\right) \leq 1$, one easily has

$$
\begin{aligned}
\left|I_{4}^{1}\right| \leq & \int_{\left\{\left|u_{n}\right|<k\right\}} c(x, t)\left|\varphi\left(T_{k}\left(u_{n}\right)-T_{k}(u)_{\mu}\right)\right| \\
& +\frac{b(k)}{\alpha} \int_{\left\{\left|u_{n}\right|<k\right\}}\left|\nabla u_{n}\right|^{p}\left|\varphi\left(T_{k}\left(u_{n}\right)-T_{k}(u)_{\mu}\right)\right| \\
\leq & \frac{b(k)}{\alpha} \int_{Q}\left[a\left(x, t, \nabla T_{k}\left(u_{n}\right)\right)-a\left(x, t, \nabla T_{k}(u)_{\mu}\right)\right]\left[\nabla T_{k}\left(u_{n}\right)-\nabla T_{k}(u)_{\mu}\right] \\
& \times\left|\varphi\left(T_{k}\left(u_{n}\right)-T_{k}(u)_{\mu}\right)\right| d x d t+\epsilon(n, \mu),
\end{aligned}
$$

and also we have the same estimation of $I_{4}^{2}$.

Then

$$
\begin{aligned}
\left|I_{4}^{1}\right|+\left|I_{4}^{2}\right| \leq & 2 \frac{b(k)}{\alpha} \int_{Q}\left[a\left(x, t, \nabla T_{k}\left(u_{n}\right)\right)-a\left(x, t, \nabla T_{k}(u)_{\mu}\right)\right]\left[\nabla T_{k}\left(u_{n}\right)-\nabla T_{k}(u)_{\mu}\right] \\
& \times\left|\varphi\left(T_{k}\left(u_{n}\right)-T_{k}(u)_{\mu}\right)\right| d x d t+\epsilon(n, \mu) .
\end{aligned}
$$


14 Parabolic inequalities in $L^{1}$

By denoting by $J_{1}$ the third term of (2.45), one can write

$$
\begin{aligned}
& J_{1}= \int_{Q} a\left(x, t, \nabla u_{n}\right)\left(\nabla T_{k}\left(H_{m}\left(u_{n}\right)\right)-\nabla T_{k}\left(H_{m}(u)\right)_{\mu}\right) \\
& \times h_{m}\left(u_{n}\right) \rho_{l}\left(H_{m}\left(u_{n}\right)\right) \varphi^{\prime}\left(T_{k}\left(H_{m}\left(u_{n}\right)\right)-T_{k}\left(H_{m}(u)\right)_{\mu}\right) d x d t \\
&= \int_{Q} a\left(x, t, \nabla T_{k}\left(u_{n}\right)\right)\left(\nabla T_{k}\left(H_{m}\left(u_{n}\right)\right)-\nabla T_{k}\left(H_{m}(u)\right)_{\mu}\right) h_{m}\left(u_{n}\right) \\
& \times \rho_{l}\left(H_{m}\left(u_{n}\right)\right) \varphi^{\prime}\left(T_{k}\left(H_{m}\left(u_{n}\right)\right)-T_{k}\left(H_{m}(u)\right)_{\mu}\right) d x d t \\
&+\int_{\left\{\left|u_{n}\right|>k\right\}} a\left(x, t, \nabla u_{n}\right)\left(\nabla T_{k}\left(H_{m}\left(u_{n}\right)\right)-\nabla T_{k}\left(H_{m}(u)\right)_{\mu}\right) h_{m}\left(u_{n}\right) \\
& \quad \times \rho_{l}\left(H_{m}\left(u_{n}\right)\right) \varphi^{\prime}\left(T_{k}\left(H_{m}\left(u_{n}\right)\right)-T_{k}\left(H_{m}(u)\right)_{\mu}\right) d x d t, \\
& J_{1}=\int_{Q} a\left(x, t, \nabla T_{k}\left(u_{n}\right)\right)\left(\nabla T_{k}\left(u_{n}\right)-\nabla T_{k}(u)_{\mu}\right) \rho_{l}\left(u_{n}\right) \varphi^{\prime}\left(T_{k}\left(u_{n}\right)-T_{k}(u)_{\mu}\right) d x d t \\
& \quad-\int_{\left\{\left|u_{n}\right|>k\right\}} \rho_{l}\left(u_{n}\right) a\left(x, t, \nabla u_{n}\right)\left(\nabla T_{k}(u)_{\mu}\right) \varphi^{\prime}\left(T_{k}\left(u_{n}\right)-T_{k}(u)_{\mu}\right) d x d t+\epsilon(m) .
\end{aligned}
$$

Since $a\left(x, t, \nabla u_{n}\right) \rho_{l}\left(u_{n}\right)$ is bounded in $L^{p^{\prime}}(Q)$, we deduce that

$$
a\left(x, t, \nabla u_{n}\right) \rho_{l}\left(u_{n}\right) \longrightarrow a(x, t, \nabla u) \rho_{l}(u) \quad \text { weakly in } L^{p^{\prime}}(Q),
$$

and so

$$
\begin{aligned}
J_{1}=\int_{Q} & \left(a\left(x, t, \nabla T_{k}\left(u_{n}\right)\right)-a\left(x, t, \nabla T_{k}(u)_{\mu}\right)\right)\left(\nabla T_{k}\left(u_{n}\right)-\nabla T_{k}(u)_{\mu}\right) \\
& \times \varphi^{\prime}\left(T_{k}\left(u_{n}\right)-T_{k}(u)_{\mu}\right) d x d t+\epsilon(m, n, \mu) .
\end{aligned}
$$

Concerning the second term of (2.45), one easily has

$$
\begin{aligned}
& \int_{Q} a\left(x, t, \nabla u_{n}\right) \nabla u_{n} h_{m}^{2}\left(u_{n}\right) \rho_{l}^{\prime}\left(H_{m}\left(u_{n}\right)\right) \varphi\left(T_{k}\left(H_{m}\left(u_{n}\right)\right)-\omega_{\mu}^{m}\right) d x d t \\
& \quad \leq \varphi(2 k) \int_{\left\{l \leq\left|u_{n}\right|<l+1\right\}} a\left(x, t, \nabla u_{n}\right) \nabla u_{n} d x d t
\end{aligned}
$$

and since

$$
\int_{\left\{l \leq\left|u_{n}\right|<l+1\right\}} a\left(x, t, \nabla u_{n}\right) \nabla u_{n} d x d t \leq \int_{\left|u_{n}\right|>l}|f| d x d t
$$


we deduce that

$$
\begin{aligned}
& \left|\int_{Q} a\left(x, t, \nabla u_{n}\right) \nabla u_{n} h_{m}^{2}\left(u_{n}\right) \rho_{l}^{\prime}\left(H_{m}\left(u_{n}\right)\right) \varphi\left(T_{k}\left(H_{m}\left(u_{n}\right)\right)-\omega_{\mu}^{m}\right) d x d t\right| \\
& \quad \leq \varphi(2 k) \int_{\left|u_{n}\right|>l}|f| d x d t=\epsilon(n, l) .
\end{aligned}
$$

The same result can be obtained for the fourth term of (2.45).

Combining (2.45)-(2.65), using the fact that $\phi^{\prime}-2(b(k) / \alpha)|\phi| \geq 1 / 2$ for $\delta \geq$ $(b(k) / \alpha)^{2}$, we deduce that

$$
\lim _{n \rightarrow \infty} \int_{Q}\left[a\left(x, t, \nabla T_{k}\left(u_{n}\right)\right)-a\left(x, t, \nabla T_{k}(u)_{\mu}\right)\right]\left[\nabla T_{k}\left(u_{n}\right)-\nabla T_{k}(u)_{\mu}\right] d x d t=0 .
$$

On the other hand, we have

$$
\begin{aligned}
& \int_{Q}\left[a\left(x, t, \nabla T_{k}\left(u_{n}\right)\right)-a\left(x, t, \nabla T_{k}(u)\right)\right]\left[\nabla T_{k}\left(u_{n}\right)-\nabla T_{k}(u)\right] d x d t \\
& \quad-\int_{Q}\left[a\left(x, t, \nabla T_{k}\left(u_{n}\right)\right)-a\left(x, t, \nabla T_{k}(u)_{\mu}\right)\right]\left[\nabla T_{k}\left(u_{n}\right)-\nabla T_{k}(u)_{\mu}\right] d x d t \\
& =\int_{Q} a\left(x, t, \nabla T_{k}\left(u_{n}\right)\right)\left(\nabla T_{k}(u)_{\mu}-\nabla T_{k}(u)\right) d x d t \\
& \quad-\int_{Q} a\left(x, t, \nabla T_{k}(u)\right)\left(\nabla T_{k}\left(u_{n}\right)-\nabla T_{k}(u)\right) d x d t \\
& \quad+\int_{Q} a\left(x, t, \nabla T_{k}(u)_{\mu}\right)\left(\nabla T_{k}\left(u_{n}\right)-\nabla T_{k}(u)_{\mu}\right) d x d t=\epsilon(n, \mu) .
\end{aligned}
$$

Consequently by [1, Lemma 5], we obtain

$$
T_{k}\left(u_{n}\right) \longrightarrow T_{k}(u) \quad \text { strongly in } L^{p}\left(0, T ; W_{0}^{1, p}(\Omega)\right) \text { for every } k>0 .
$$

Step 5 (passage to the limit). Letting $v \in D \cap \mathscr{H} \cap L^{\infty}(Q)$, and using $T_{k}\left(H_{m}\left(u_{n}\right)-\theta v\right)$ as test function in the problem $\left(R_{n}\right)$, we obtain

$$
\begin{aligned}
& \int_{0}^{T}\left\langle\frac{\partial H_{m}\left(u_{n}\right)}{\partial t}, T_{k}\left(H_{m}\left(u_{n}\right)-\theta v\right)\right\rangle d t+\int_{Q} a\left(x, t, \nabla u_{n}\right) \nabla T_{k}\left(H_{m}\left(u_{n}\right)-\theta v\right) h_{m}\left(u_{n}\right) d x d t \\
& \quad+\int_{Q} a\left(x, t, \nabla u_{n}\right) \nabla u_{n} T_{k}\left(H_{m}\left(u_{n}\right)-\theta v\right) h_{m}^{\prime}\left(u_{n}\right) d x d t \\
& \quad+\int_{Q}\left|h\left(x, u_{n}\right)\right|^{n-1} h\left(x, u_{n}\right)\left|G\left(x, t, u_{n}, \nabla u_{n}\right)\right| h_{m}\left(u_{n}\right) T_{k}\left(H_{m}\left(u_{n}\right)-\theta v\right) d x d t \\
& \leq \int_{Q} f T_{k}\left(H_{m}\left(u_{n}\right)-\theta v\right) h_{m}\left(u_{n}\right) d x d t .
\end{aligned}
$$


We have

$$
\begin{aligned}
\int_{Q}\left|h\left(x, u_{n}\right)\right|^{n-1} h\left(x, u_{n}\right)\left|G\left(x, t, u_{n}, \nabla u_{n}\right)\right| h_{m}\left(u_{n}\right) T_{k}\left(H_{m}\left(u_{n}\right)-\theta v\right) d x d t \\
\geq \int_{\left\{0 \leq H_{m}\left(u_{n}\right) \leq \theta v\right\}}\left|h\left(x, u_{n}\right)\right|^{n-1} \\
\quad \times h\left(x, u_{n}\right)\left|G\left(x, t, u_{n}, \nabla u_{n}\right)\right| h_{m}\left(u_{n}\right) T_{k}\left(H_{m}\left(u_{n}\right)-\theta v\right) d x d t \\
\quad+\int_{\left\{\theta v \leq H_{m}\left(u_{n}\right) \leq 0\right\}}\left|h\left(x, u_{n}\right)\right|^{n-1} \\
\quad \times h\left(x, u_{n}\right)\left|G\left(x, t, u_{n}, \nabla u_{n}\right)\right| h_{m}\left(u_{n}\right) T_{k}\left(H_{m}\left(u_{n}\right)-\theta v\right) d x d t .
\end{aligned}
$$

Now we deal with the estimation of the last two terms in the right-hand side of the last inequality which we denote, respectively, by $J_{1}^{\prime}(m, n)$ and $J_{2}^{\prime}(m, n)$. Let us define

$$
\delta_{1}(x, t)=\sup _{0 \leq s \leq \theta v} h(x, s)
$$

then we get $0 \leq \delta_{1}(x, t)<1$ a.e. in $Q$.

We have

$$
\begin{aligned}
\limsup _{m \rightarrow \infty}\left|J_{1}^{\prime}(m, n)\right| & \leq k \int_{\left\{0 \leq u_{n} \leq \theta v\right\}}(\delta(x, t))^{n}\left(c(x, t)+\left|\nabla u_{n}\right|^{p}\right) \\
& \leq \int_{\left\{\left|u_{n}\right| \leq\|v\|_{\infty}\right\}}(\delta(x, t))^{n}\left(c(x, t)+\left|\nabla u_{n}\right|^{p}\right),
\end{aligned}
$$

and by using the strong convergence of $T_{\|v\|_{\infty}}\left(u_{n}\right)$ in $L^{p}\left(0, T ; W_{0}^{1, p}(\Omega)\right)$, we deduce that

$$
\limsup _{n \rightarrow \infty} \limsup _{m \rightarrow \infty}\left|J_{1}^{\prime}(m, n)\right|=0
$$

with the same technique (taking $\delta_{2}(x, t)=\inf _{\theta v \leq s \leq 0} h(x, s)$ ), we can see that

$$
\limsup _{m \rightarrow \infty}\left|J_{2}^{\prime}(n, m)\right| \longrightarrow 0 \quad \text { as } n \longrightarrow+\infty \text {. }
$$

On the other hand,

$$
\begin{aligned}
\int_{Q} a\left(x, t, \nabla u_{n}\right) \nabla T_{k}\left(H_{m}\left(u_{n}\right)-\theta v\right) h_{m}\left(u_{n}\right) d x d t \\
=\int_{Q} a\left(x, t, \nabla u_{n}\right) \nabla\left(H_{m}\left(u_{n}\right)-\theta v\right) \chi_{\left\{\left|H_{m}\left(u_{n}\right)-\theta v\right| \leq k\right\}} h_{m}\left(u_{n}\right) d x d t \\
=\int_{Q}\left(a\left(x, t, \nabla u_{n}\right)-a(x, t, \theta \nabla v)\right) \nabla\left(H_{m}\left(u_{n}\right)-\theta v\right) \chi_{\left\{\left|H_{m}\left(u_{n}\right)-\theta v\right| \leq k\right\}} h_{m}\left(u_{n}\right) d x d t \\
\quad+\int_{Q} a(x, t, \theta \nabla v) \nabla\left(H_{m}\left(u_{n}\right)-\theta v\right) \chi_{\left\{\left|H_{m}\left(u_{n}\right)-\theta v\right| \leq k\right\}} h_{m}\left(u_{n}\right) d x d t .
\end{aligned}
$$


Since $a(x, t, \theta v)$ belongs to $\left(L^{p^{\prime}}(Q)\right)^{N}$, using Fatou's lemma in the first term of the last side gives

$$
\liminf _{n, m \rightarrow+\infty} \int_{0}^{T}\left\langle A u_{n}, T_{k}\left(H_{m}\left(u_{n}\right)-\theta v\right)\right\rangle d t \geq \int_{0}^{T}\left\langle A u, T_{k}(u)-\theta v\right\rangle d t
$$

Go back to (2.69) and pass to the limit as $m, n \rightarrow \infty$ to obtain

$$
\int_{0}^{T}\left\langle\theta \frac{\partial v}{\partial t}, T_{k}(u-\theta \nabla v)\right\rangle d t+\int_{Q} a(x, t, \nabla u) \nabla T_{k}(u-\theta v) d x d t \leq \int_{Q} f T_{k}(u-\theta v) d x d t
$$

Letting now $\theta$ tend to 1 , we get

$$
\int_{0}^{T}\left\langle\frac{\partial v}{\partial t}, T_{k}(u-\theta v)\right\rangle d t+\int_{Q} a(x, t, \nabla u) \nabla T_{k}(u-v) d x d t \leq \int_{Q} f T_{k}(u-v) d x d t,
$$

which completes the proof.

Remark 2.4. The same technique allows us to prove an existence result for solutions of the following parabolic inequalities:

$$
\begin{gathered}
q_{-}(x) \leq u(x, t) \leq q_{+}(x) \text { a.e. in } Q, \\
T_{k}(u) \in L^{p}\left(0, T ; W_{0}^{1, p}(\Omega)\right), \\
\int_{0}^{T}\left\langle\frac{\partial v}{\partial t}, T_{k}(u-v)\right\rangle d t+\int_{Q} a(x, t, \nabla u) \nabla T_{k}(u-v) d x d t+\int_{Q} H(x, t, u, \nabla u) T_{k}(u-v) d x d t \\
\leq \int_{Q} f T_{k}(u-v) d x d t, \quad \forall v \in \mathscr{K} \cap D \cap L^{\infty}(Q),
\end{gathered}
$$

where $H$ is a given Carathéodory function satisfying, for all $(s, \zeta) \in \mathbb{R} \times \mathbb{R}^{N}$ and a.e. $(x, t) \in Q$, the following conditions:

$$
\begin{gathered}
|H(x, t, s, \zeta)| \leq \lambda(|s|)\left(\delta(x, t)+|\zeta|^{p}\right), \\
H(x, t, s, \zeta) s \geq 0
\end{gathered}
$$

with $\lambda: \mathbb{R}^{+} \rightarrow \mathbb{R}^{+}$is a continuous increasing function and $\delta(x, t)$ is a given positive function in $L^{1}(Q)$.

\section{Acknowledgments}

This work has been supported by LERMA (EMI), LMA (FSR), and Volkswagen Foundation Grant no. I/79315. 


\section{References}

[1] L. Boccardo, F. Murat, and J.-P. Puel, Existence of bounded solutions for nonlinear elliptic unilateral problems, Annali di Matematica Pura ed Applicata 152 (1988), no. 1, 183-196.

[2] F. Donati, A penalty method approach to strong solutions of some nonlinear parabolic unilateral problems, Nonlinear Analysis 6 (1982), no. 6, 585-597.

[3] R. Landes, On the existence of weak solutions for quasilinear parabolic initial-boundary value problems, Proceedings of the Royal Society of Edinburgh. Section A. Mathematics 89 (1981), no. 3-4, 217-237.

[4] R. Landes and V. Mustonen, A strongly nonlinear parabolic initial-boundary value problem, Arkiv för Matematik 25 (1987), no. 1, 29-40.

[5] J.-L. Lions, Quelques méthodes de résolution des problèmes aux limites non linéaires, Dunod, Paris; Gauthier-Villars, Paris, 1969.

[6] D. Meskine and A. Elmahi, On the limit of some nonlinear parabolic problems, Archives of Inequalities and Applications 2 (2004), no. 4, 499-515.

[7] M. C. Palmeri, Homographic approximation for some nonlinear parabolic unilateral problems, Journal of Convex Analysis 7 (2000), no. 2, 353-373.

[8] A. Porretta, Existence results for nonlinear parabolic equations via strong convergence of truncations, Annali di Matematica Pura ed Applicata 177 (1999), 143-172.

[9] M. Rudd, Nonlinear constrained evolution in Banach spaces, Ph.D. thesis, University of Utah, Utah, 2003.

[10] W Weak and strong solvability of parabolic variational inequalities in Banach spaces, Journal of Evolution Equations 4 (2004), no. 4, 497-517.

[11] J. Simon, Compact sets in the space $L^{p}(0, T ; B)$, Annali di Matematica Pura ed Applicata 146 (1987), no. 1, 65-96.

K. Azelmat: LMA, GAN, Département de Mathématiques et d'Informatique, Faculté des Sciences, Université Mohammed V Agdal, Avenue Ibn Battouta, Rabat BP 1014, Morocco

E-mail address: azelmat@fsr.ac.ma

M. Kbiri Alaoui: LERMA, Ecole Mohammadia d'Ingénieurs, Université Mohammed V Agdal, Avenue Ibn Sina, Agdal, Rabat BP 765, Morocco; LMA, GAN, Département de Mathématiques et d'Informatique, Faculté des Sciences, Université Mohammed V Agdal, Avenue Ibn Battouta, Rabat BP 1014, Morocco

E-mail address: mka_la@yahoo.fr

D. Meskine: LERMA, Ecole Mohammadia d'Ingénieurs, Université Mohammed V Agdal, Avenue Ibn Sina, Agdal, Rabat BP 765, Morocco; LMA, GAN, Département de Mathématiques et d'Informatique, Faculté des Sciences, Université Mohammed V Agdal, Avenue Ibn Battouta, Rabat BP 1014, Morocco

E-mail address: driss.meskine@laposte.net

A. Souissi: LMA, GAN, Département de Mathématiques et d'Informatique, Faculté des Sciences, Université Mohammed V Agdal, Avenue Ibn Battouta, Rabat BP 1014, Morocco

E-mail address: souissi@fsr.ac.ma 


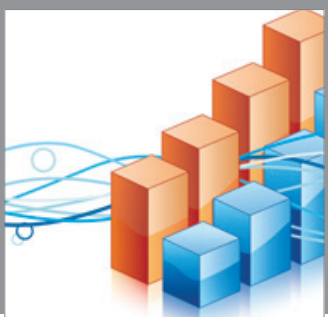

Advances in

Operations Research

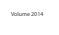

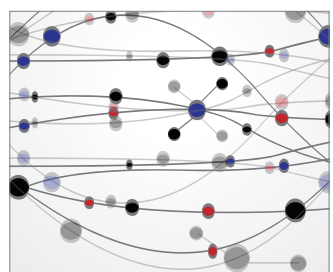

\section{The Scientific} World Journal
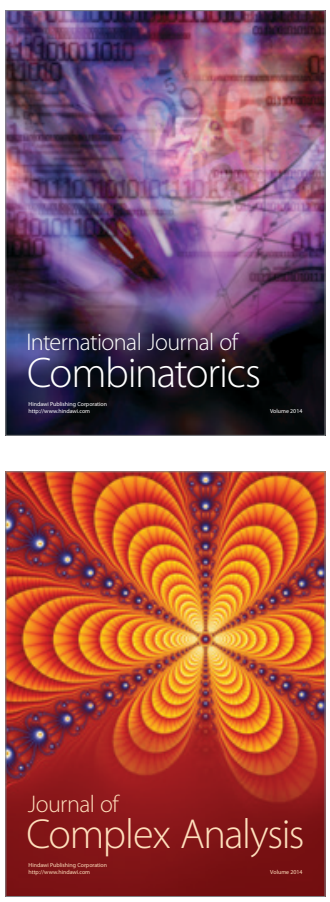

International Journal of

Mathematics and

Mathematical

Sciences
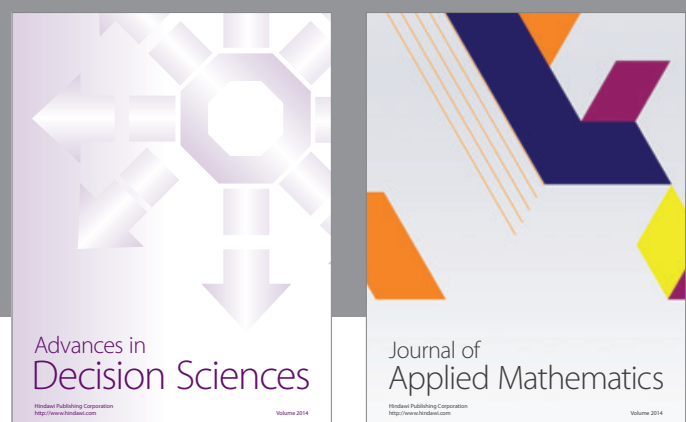

Journal of

Applied Mathematics
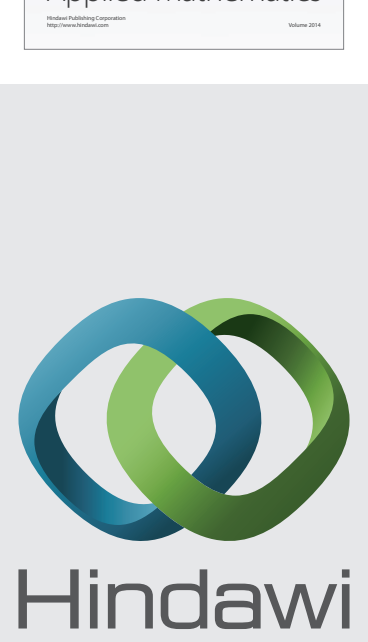

Submit your manuscripts at http://www.hindawi.com
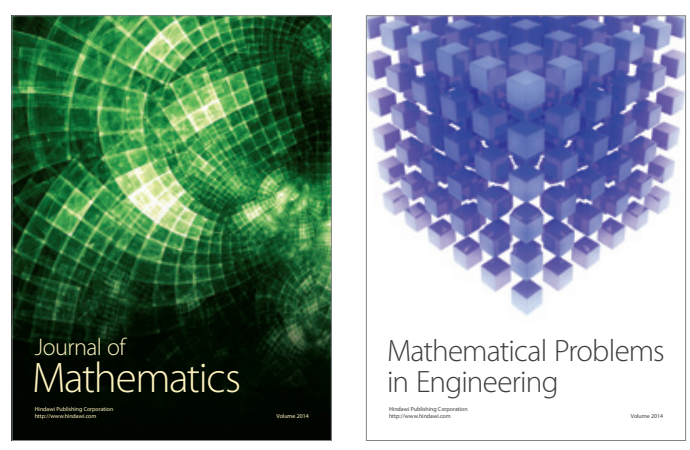

Mathematical Problems in Engineering
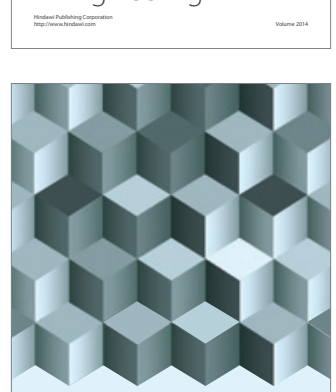

Journal of

Function Spaces
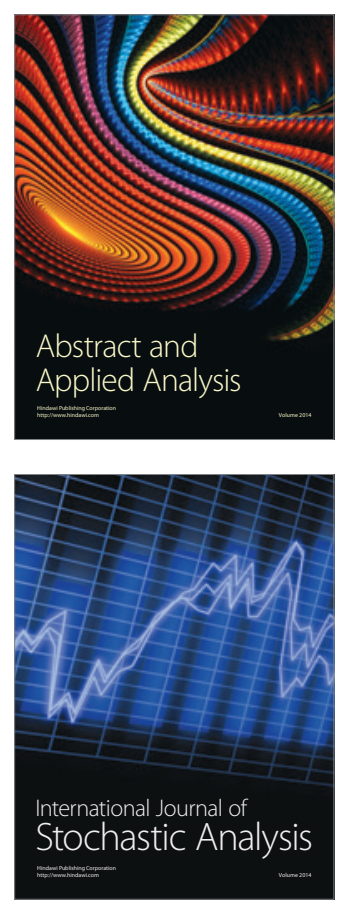

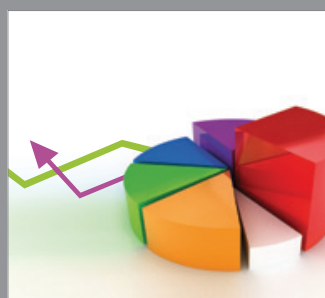

ournal of

Probability and Statistics

Promensencen
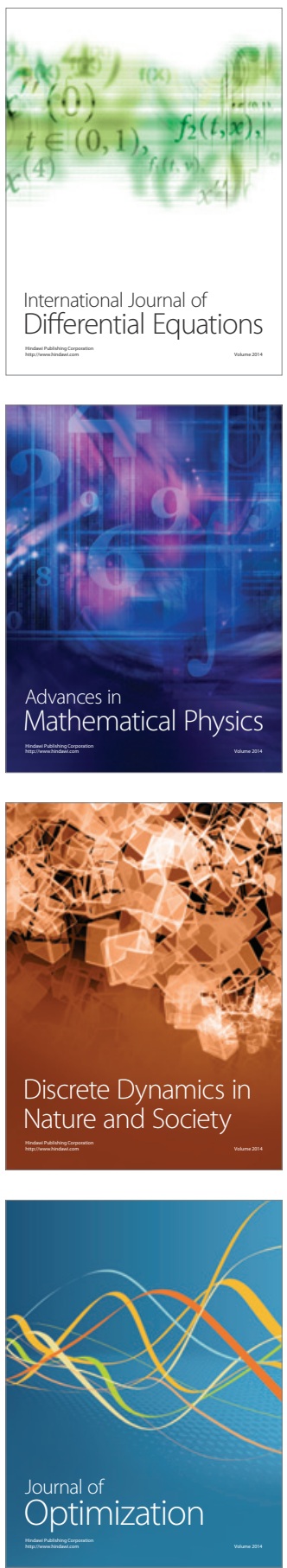